\title{
《地球物理学报》编辑委员会
}

\section{Editorial Committee of Chinese Journal of Geophysics}

主 编 (Editor-in-Chief)

常务副主编 (Executive Deputy Editor-in-Chief)

副主编 (Deputy Editors-in-Chief)

汪毓明 WANG YuMing

陈运泰 CHEN YunTa

潘永信 PAN YongXin

刘 洪 LIU Hong

编 委 (Editorial Board Members)

艾印双 AI YinShuang
底青云 DI QingYun
胡祥云 HU XiangYun
金振民 JIN ZhenMin
刘立波 LIU LiBo
吕达仁 LU DaRen
孙和平 SUN HePing
王家林 WANG JiaLin
吴忠良 WU ZhongLiang
杨长春 YANG ChangChun
郑天愉 ZHENG TianYu
Ivan PSENCIK
Rushan WU(吴如山)
朱日祥 ZHU RiXiang
常 旭 CHANG Xu 符力耘 FU LiYun 黄宝春 HUANG BaoChun 李 斐 LI Fei

刘启元 LIU QiYuan 倪四道 NI SiDao 孙建国 SUN JianGuo 王西文 WANG XiWen 徐继生 XU JiSheng 杨文采 YANG WenCai Longsang CHAN(陈龙生) Jerming CHIU(邱哲明) Xiaobi XIE(谢小碧)
陈棋福 CHEN QiFu 高静怀 GAO JingHuai 黄金水 HUANG JinShui 李幼铭 LI YouMing 刘瑞源 LIU RuiYuan 沈正康 SHEN ZhengKang 万卫星 WAN WeiXing 王绪本 WANG XuBen 徐文耀 XU WenYao 姚振兴 YAO ZhenXing Henry Pollack Kunhua CHEN(陈昆华) Yu ZHANG(张宇)
陈晓非 CHEN XiaoFei 何丽娟 HE LiJuan 黄忠贤 HUANG ZhongXian 刘代志 LIU DaiZhi 刘树华 LIU ShuHua 石耀霖 SHI YaoLin 王椿镛 WANG ChunYong 魏文博 WEI WenBo 杨宝俊 YANG BaoJun 张剑锋 ZHANG JianFeng Huawei ZHOU (周华伟) T. Yanovskaya

\section{地球物理学表}

\section{DIQIU WULI XUEBAO(月刊 1948 年创刊)} 第 63 卷 第 9 期 2020 年 9 月

\section{CHINESE JOURNAL OF GEOPHYSICS}

(Monthly, Started in 1948)

Vol. 63 No.9 September, 2020

\section{主管单 位 \\ 中国科学院}

主 办

编

辑

中国科学院地质与地球物理研究所 中国 地球 物 理 学 会 地球物理学报编辑委员会

地址: 北京 y825 信箱 那政编码: 10002y

电话:010-82998105

网址 : http://www. geophy. cn

E-mail:actageop@mail.igcas.ac.cn

主

出

印刷装订 总发 行

国外发行
潘永信

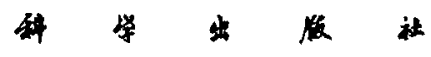

北京科信印刷有限公司

\section{甜㘶出版社 \\ 地址: 北京东黄城根北街 16 号 邮政编码: 100717 \\ 电话: $010-64017032$ \\ E-mail:journal@mail.sciencep.com}

地址: 北京 399 信箱 那政编码: 100044

Superintended by Chinese Academy of Sciences

Sponsored by Institute of Geology and Geophysics, CAS Chinese Geophysical Society

Edited by Editorial Board of Chinese

Journal of Geophysics

Add: P. O. Box 9825. Beijing 100029. China

Tel:010-82998105

Web: http://www. geophy, cn

E-mail :actageop@mail.igcas.ac.cn

Editor-in-Chief PAN YongXin

Published by Science Press

Printed by Beijing Kexin Printing Co., Ltd

Distributed by Science Press

Add:16 Donghuangchenggen North Street,

Beijing 100717 , China

Tel:010-64017032

E-mail: journal@mail. sciencep.com

Foreign China Intemational Book Jrading Corporation Add: P. O. Box 399, Beijing 100044, China

国内统一刊号:C.N 11-2074/P 国内邮发代号:2-571 国外发行代号:BM 76 定价:120.00 元 国内外公开发行

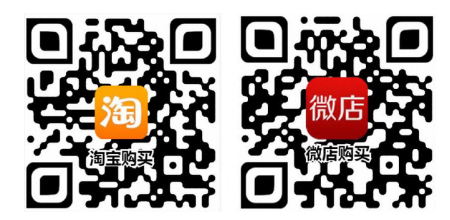

I S SN 0001-5733

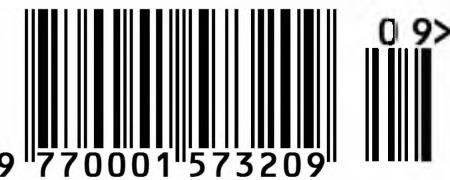

